\title{
Clinico-Epidemiological Study of Acute Flaccid Paralysis at a Tertiary Centre
}

\author{
Chaudhary $\mathrm{S}^{1}$, Bhatta $\mathrm{NK}^{2}$, Khanal $\mathrm{B}^{3}$, Bhandari $\mathrm{R}^{4}$, Singh $\mathrm{RR}^{5}$
}

\begin{abstract}
Introduction: Acute Flaccid Paralysis (AFP) can be caused by various causes including poliomyelitis and sometimes even Acute Encephalitis Syndrome (AES). The objective of this study was to study the clinico-epidemiological profile of different causes of AFP including AES and their associated co-morbidities. Materials and Methods: A prospective hospital-based study was carried out including all cases fulfilling AFP case definition. History, clinical examination, necessary investigations were performed and required treatment given. Regular follow-ups were done and final classification made alongwith AFP surveillance team. Results: Out of 43 children included in the study, 18 expired and 25 completed follow-up. Final classification showed 53.5\% AES, 9.3\% Guillain Barre Syndrome (GBS), 9.3\% dyselectrolytemia, 9.3\%, peripheral neuritis, $7 \%$ Non-Polio Entero Virus (NPEV) and $11.6 \%$ others. Fever, altered sensorium and convulsions were present in $79.1 \%, 65.1 \%$ and $58.1 \%$ respectively. Eightyfour percent had asymmetrical paralysis with quadriparesis in $72 \%$. Cerebrospinal fluid was abnormal in $34.9 \%$. Japanese encephalitis serology was positive in $4.7 \%$. The mean GCS was $9.53 \pm 4.27$ with a significant difference between survivors and non-survivors $(p=0.02)$. Almost half $(52.2 \%)$ required ICU care, of which 14 expired ( $p=0.005)$. Seventeen children needed mechanical ventilation, of which 13 died $(p<0.001)$. Requirement of inotropes and complications like respiratory failure and autonomic failure were significantly related to death. Conclusion: AES, being one of the commonest causes of AFP, should be included in AFP surveillance. Poor GCS, requirement of inotropes, complications like respiratory failure and autonomic failure are related with poor prognosis.
\end{abstract}

Key words: Acute Encephalitis Syndrome (AES), Acute Flaccid Paralysis (AFP), Guillain Barre Syndrome (GBS), Japanese Encephalitis (JE)

\section{Introduction}

A cute flaccid paralysis (AFP), as defined by World Health Organization (WHO), is sudden onset of weakness and floppiness in any part of the body in a child less than 15 years age or if a clinician suspects polio in any person of any age (excluding adults, spastic paralysis, old cases or cases with obvious causes like trauma ${ }^{1,2}$.
'Dr. Shipra Chaudhary, MBBS, MD, Resident, Department of Paediatrics and Adolescent Medicine, ${ }^{2}$ Dr. Nisha Keshary Bhatta, MBBS, $M D$, Professor, Department of Paediatrics and Adolescent Medicine. ${ }^{3}$ Dr. Basudha Khanal, MBBS, $M D$, Professor, Department of Microbiology, ${ }^{4} \mathrm{Dr}$. Rabin Bhandari, MBBS, MD, Associate Professor, Department of General Practice and Emergency Medicine, ${ }^{5} \mathrm{Dr}$. Rupa Rajbhandari Singh, MBBS, $M D$, Professor, Department of Paediatrics and Adolescent Medicine. All from BP Koiral Instutute of Health Sciences, Dharan, Nepal.

\section{Address for correspondence:}

Dr. Shipra Chaudhary, Senior Resident, Department of Paediatrics and Adolescent Medicine,

BP Koirala Institute of Health Sciences, Dharan, Nepal.

E-mail: shiprachaudhary@hotmail.com

\section{How to cite}

Chaudhary S, Bhatta NK, Khanal B, Bhandari R, Singh RR. Clinico-Epidemiological Study of Acute Flaccid Paralysis at a Tertiary Centre. J Nepal Paediatr Soc 2014;34(2):104-110.

doi: http://dx.doi.org/10.3126/jnps.v34i2.9794

This work is licensed under a Creative Commons Attribution 3.0 License.

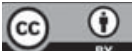

There are many conditions leading to AFP, poliomyelitis being one of the most important differential diagnoses. The diagnoses most often confused with polio are Guillain-Barre syndrome (GBS), transverse myelitis, traumatic paralysis and sometimes other neurotropic viruses like 
Rabies, Japanese encephalitis. Similarly, critical illnesses like polyneuropathy, metabolic disorder like hypokalemic periodic paralysis, polymyositis, neuropathies, neuromuscular junction disorders and tumours should also be considered in evaluation of $\mathrm{AFP}^{3}$.

The possibility of polio should be considered in any case of AFP ${ }^{4}$. Polio eradication is based on WHO's four- pronged eradication strategy: 1 ) routine immunization with oral polio vaccine (OPV); 2) supplementary, additional doses of OPV countrywide during National Immunization Days (NIDs); 3) moppingup immunization activities to catch any below five children who may have been missed during stages one and two; and 4) enhanced surveillance for all cases of AFP and wild poliovirus ${ }^{3}$. WHO has established standards to check the efficacy of surveillance systems in each country, polio-endemic or not, should be able to detect at least two cases of AFP not caused by polio every 100,000 children under age $15^{3}$.

Globally, polio cases have decreased by over 99\% since 1988, the year Global Polio Eradication Initiative was launched ( 350000 cases; >125 endemic countries) to 2009 (1604 reported cases) ${ }^{4,5}$. In 2010, only parts of 4 countries -Pakistan, Afghanistan, Nigeria and India remained endemic for polio ${ }^{4}$. But in 2009-2010, 23 previously polio-free countries were reinfected due to imports of virus. The year 2011 saw a significant decline in the total number of polio cases world-wide. After having zero cases of wild poliovirus for a year, India is no more in the list of polio-endemic countries since January 13, 2011.

Despite the overall decline, there has been a steep increase in polio cases in 2011 in the other three polio-endemic countries ${ }^{4}$. The increase in cases in key endemic areas constitutes an incontrovertible risk to global polio eradication. As long as a single child remains infected; children in all countries remain at risk of contracting polio ${ }^{4}$. Frequently, a child with acute encephalitis syndrome may have acute flaccid paralysis which also should be investigated and not a single case of AFP should go unevaluated. Acute Encephalitis Syndrome (AES) surveillance has also been integrated with AFP surveillance since $2004^{6,7}$.

The AFP surveillance in Nepal was initiated in 1995 with passive reporting of AFP cases through the Early Warning and Reporting System (EWARS), a sentinel system for surveillance of six priority infectious diseases $3,4,5$. Nepal has been free of indigenous poliovirus since 2000. However, due to its proximity to India and sociocultural relations, it has been subjected to repeated polio importations, in particular from Bihar ${ }^{3}$. After having zero case record for four years from 2001 to 2004; it faced resurgence of polio in 2005 when four cases of wild polio were detected in the terai region. According to the AFP surveillance record, there were 451 cases in 2009, 564 in 2010 and 567 in 2011 amongst which, there were no cases of polio in 2009, 6 polio cases in $2010^{5}$ and none detected since 2011. Nepal will continue to be at particular risk of re-infection until all endemic polio transmission in neighbouring countries has been successfully interrupted.

According to B P Koirala Institute of Health Sciences (BPKIHS) hospital statistics, there were a very few cases of AFP reported from 2008 to 2010, with no cases of wild polio, and the majority being Guillain Barre Syndrome.

As Nepal faced re-emergence of polio in the last six years period, and BPKIHS being referral as well as focal centre of EWARS in Eastern region, we felt the need to take up this study. This study not only helped in finding out the clinico-epidemiological pattern of AFP in the Eastern Nepal but also supported in the polio surveillance in-depth as no case would go unreported when we involved all the differential diagnoses including AES.

Objectives: 1) To study the clinico-epidemiological profile of the differential diagnoses of Acute Flaccid Paralysis (AFP) including AES with flaccid paralysis, 2) To study the associated co-morbidities in children with AFP.

\section{Materials and Methods}

It was a prospective hospital-based study done over one year period in 2011 in the Department of Paediatrics and Adolescent Medicine, BPKIHS, Dharan, Nepal.

Inclusion Criteria: Any child of age $\leq 15$ years presenting with Acute Flaccid Paralysis (AFP) in any form including acute encephalopathies or Acute Encephalitis Syndrome (AES) as defined by WHO.

Exclusion Criteria: Age $>15 y r s$, spastic paralysis, old cases or cases with obvious causes like trauma, obvious congenital anomalies or birth defects, AES without acute flaccid paralysis.

Sample size: All cases fulfilling the inclusion criteria during the study period were included in the study.

After taking consent from the parent/guardian, all children fulfilling inclusion criteria were enrolled and detail history with clinical examination were recorded 
in a pre-designed pro forma. The relevant laboratory and/or radiological investigations were carried out like complete blood counts; electrolytes, blood cultures; rapid diagnostic test for malaria screening. Cerebrospinal Fluid (CSF) was collected and sent for cytology, biochemistry, microscopy and culture for bacteriology. Test for antibody to JE virus in CSF and serum was done by IgM capture ELISA at JE laboratory, BPKIHS. Stool samples were sent for examination as per AFP surveillance guidelines and sent to the IPD field office and then to the WHO-accredited Poliovirus Laboratory in Bangkok, Thailand.

Based on detail clinical evaluation, relevant investigations and WHO case definitions, diagnoses were made and treatment instituted. Patients were observed for any complications or sequelae. Regular follow-ups were done after discharge at 15 days/30 days/60 days alongwith the surveillance team and final classification made.

The acquired data were entered in SPSS Statistics software version 14. Differences between clinical groups (survivors and non-survivors) were compared using Student independent samples t-tests (for normally distributed data), Mann-Whitney U-tests (for non-normally distributed data) and Fisher Exact tests (for categorical data/proportions). Statistical significance was set at the conventional $5 \%$ level for all analyses and final inferences made.

\section{Results}

Out of the 3432 children admitted in paediatric wards during the one-year study period, 43 (1.25\%) children were diagnosed to have AFP. The final classification showed Acute Encephalitis Syndrome (AES) in 23 (53.5\%) children. Guillain Barre Syndrome (GBS), Dyselectrolytemia and Peripheral neuritis in 4 (9.3\%)children each; Non-Polio Enterovirus in 3 (7\%) children and other causes of AFP like TB cervical spine, Neurocysticercosis (NCC), Inborn Error of Metabolism (IEM),etc. in 5 (11.6\%) children.

Maximum (37.2\%) children fell in the age group $1-5$ yrs with a mean age of $5.6 \pm 4.1$ years. Most of them were male $(58.1 \%)$ and mostly (88.4\%) Hindus. Majority came from the Eastern part of Nepal along with a few cases from Bihar, India. The duration of illness prior to hospital admission ranged from $<1$ to $>15$ days with mean duration of illness being $6.79 \pm 6.68$ days.

The important presenting features were fever (79.1\%), convulsions $(58.1 \%)$, altered mental status $(65.1 \%)$, loss of consciousness $(23.3 \%)$, headache
(30.2\%), vomiting (27.9\%), bowel/bladder involvement (27.9\%), sensation loss (11.6\%), neck pain and preceeding viral illness in $9.3 \%$ each. Paralysis was symmetrical in $36(83.7 \%)$ children and asymmetrical in $7(16.3 \%)$ children.

On clinical examination, the Glasgow Coma Scale (GCS) was found to be 3-8 in majority of the children $(44.2 \%)$. The mean GCS was $9.53 \pm 4.27$. Thirty-four (79.1\%) children had fever, out of which $20.6 \%$ had high grade temperature $>101^{\circ} \mathrm{F}$. There was associated undernutrition and pallor in $62.8 \%$ and $27.9 \%$ children respectively.

On neurological examination, mental status was found to be abnormal in 28 (65\%) children with altered sensorium in $46 \%$ and unconsciousness in $19 \%$ children. Deep Tendon Reflexes(DTR) were absent in $46.5 \%$, sluggish in $41.9 \%$ and normal in $11.6 \%$ children with AES. The signs of meningeal irritation were found in $9.3 \%$ children. Majority, i.e. 31 children with AFP (72\%) had quadriparesis, 7 paraparesis, 2 hemiparesis and 3 monoparesis. Cranial nerve involvement was seen in $7 \%$ children, all having bilateral involvement and mostly sixth nerve involvement.

One child with AES presenting with AFP was clinically diagnosed to have cerebral malaria.

There was dyselectrolytemia in the form of hypokalemia in $9.3 \%$ children with AFP. Out of the 31 children who underwent lumbar puncture, $48.4 \%$ had abnormal findings. There were 2 children with positive test for JE virus in CSF and serum (4.7\%). CT scan- head was done in 13 children, out of which $18.6 \%$ children had abnormal findings of temporal lobe cerebral abscess, thalamic infarct with frontal lobe calcification, neurocysticercosis, etc.

Stool examination was done as per AFP surveillance criteria. In 8 (18.6\%) children who expired, stool could not be collected due to very poor GCS. Among the rest 35 patients, 7\% had Non-Polio Enterovirus(NPEV) while no virus was isolated in $74.4 \%$.

Twenty-two (51.2\%) children required ICU care, out of which $39.5 \%$ received mechanical ventilation. Antibiotics were used in almost all cases- 95.3\%. Inotropes were required in $67.4 \%$ of the sick children, anticonvulsants in $60.5 \%$, osmotic diuretic in $39.5 \%$ and steroids in $25.6 \%$.

The duration of hospital stay varied within a range of 1 to 15 days with the mean stay of $6.85 \pm 4.78$ days. Twenty-three (53.5\%) children were discharged while 13 (30.2\%) went on LAMA (Left Against Medical Advice) and 
7 (16.3\%) expired. During the 60-day follow-up, 11 of the 13 children who went on LAMA expired while 2 had static course. Thus, a total of $18(41.8 \%)$ children expired and $23(53.5 \%)$ had complete recovery. Complications were observed in $21(48.8 \%)$ children in form of respiratory failure $(44.2 \%)$, autonomic failure $(27.9 \%)$, aspiration pneumonitis(14\%), acute renal failure (7\%) and undernutrition (16.3\%).

Differences between clinical groups (survivors and non-survivors) were compared using Student independent samples t-tests (for normally distributed data), Mann-Whitney U-tests (for non-normally distributed data) and Fisher Exact tests (for categorical data/proportions). On comparing the children with AFP in terms of mortality, various clinical parameters were found to be related to poor prognosis as shown in table 1 . The categorical data being binomial with small sample size were compared using Fisher Exact tests. The numerical data with Gaussian distribution were compared by Student independent samples t-tests and non-parametric data by Mann-Whitney U-tests as the two groups (survivors and non-survivors) were unpaired. The treatment details and outcome of children with AFP are tabulated in table 2.

Table 1: Comparison of clinical examination details in children with AFP

\begin{tabular}{|c|c|c|c|}
\hline Characteristic & Non-Survivors & Survivors & p-value \\
\hline Glasgow coma scale (Mean \pm SD) & $7.78 \pm 4.346$ & $10.8 \pm 3.808$ & 0.020 \\
\hline Eye response (Mean $\pm \mathrm{SD}$ ) & $2.44 \pm 1.381$ & $3.32 \pm 1.145$ & 0.029 \\
\hline Verbal response (Mean \pm SD) & $2.28 \pm 1.628$ & $3.2 \pm 1.826$ & 0.096 \\
\hline Motor response (Mean \pm SD) & $3 \pm 1.68$ & $4.28 \pm 1.568$ & 0.014 \\
\hline Endotracheal intubation & 13 & 4 & 0.001 \\
\hline Temperature (Mean \pm SD) & $99.367 \pm 1.14$ & $98.712 \pm 0.7$ & 0.025 \\
\hline Pulse (Mean \pm SD) & $107.89 \pm 18.065$ & $104.16 \pm 19.338$ & 0.525 \\
\hline Respiratory rate (Mean \pm SD) & $17.17 \pm 12.761$ & $25.16 \pm 14.38$ & 0.049 \\
\hline Systolic Blood Pressure (Mean \pm SD) & $99.22 \pm 17.904$ & $96 \pm 15.055$ & 0.526 \\
\hline Diastolic Blood Pressure (Mean \pm SD) & $56.39 \pm 18.218$ & $55.4 \pm 15.025$ & 0.883 \\
\hline Undernutrition & 11 & 16 & 1.000 \\
\hline $\begin{array}{l}\text { Mental status } \\
\text { Normal } \\
\text { Abnormal }\end{array}$ & $\begin{array}{c}4 \\
14\end{array}$ & $\begin{array}{l}11 \\
14\end{array}$ & 0.199 \\
\hline Cranial nerves involvement & 0 & 3 & 0.252 \\
\hline Abnormal movement & 3 & 2 & 0.634 \\
\hline
\end{tabular}

Table 2: Treatment details and outcome of patients with AFP in BPKIHS

\begin{tabular}{|c|c|c|c|}
\hline Characteristic & Non-survivors & Survivors & p-value \\
\hline Need of ICU care & 14 & 8 & 0.005 \\
\hline Mechanical ventilation & 13 & 4 & $<0.001$ \\
\hline Antibiotics & 18 & 23 & 0.502 \\
\hline Steroids & 6 & 5 & 0.526 \\
\hline Anticonvulsants & 11 & 15 & 1.00 \\
\hline Osmotic diuretic & 8 & 9 & 0.808 \\
\hline Inotropes & 16 & 13 & 0.019 \\
\hline Complication & 15 & 6 & $<0.001$ \\
\hline Bed sore & 1 & 0 & 0.419 \\
\hline Aspiration pneumonitis & 4 & 2 & 0.218 \\
\hline Respiratory failure & 15 & 4 & $<0.001$ \\
\hline Autonomic failure & 10 & 2 & 0.001 \\
\hline Acute renal failure & 2 & 1 & 0.562 \\
\hline Undernutrition & 12 & 17 & \\
\hline \multicolumn{4}{|c|}{ Outcome at discharge } \\
\hline \multirow{4}{*}{$\begin{array}{l}\text { Cured } \\
\text { Improved } \\
\text { LAMA } \\
\text { Death } \\
\end{array}$} & 0 & 8 & \multirow{4}{*}{$<0.001$} \\
\hline & 2 & 13 & \\
\hline & 9 & 4 & \\
\hline & 7 & 0 & \\
\hline Mean no of hospital days \pm SD & $3.8 \pm 3.516$ & $9.04 \pm 4.383$ & $<0.001$ \\
\hline
\end{tabular}




\section{Discussion}

In this prospective hospital-based study done at BPKIHS, AES was the most common cause of AFP in children. This is in contrast to most of the previous studies where GBS was the most common cause of AFP. Studies of AFP by McKhann GM et al. (1993), Morris AMS et al. (2003), Jalal et al. (2011) and Sharma KS et al. (2011) showed GBS as the major leading cause of AFP in children $8,12,13,14$. In this study, we had a total of only $4(9.3 \%)$ children with GBS while in a similar clinico-epidemiological study done at BPKIHS by Sharma KS et al, $90 \%$ were $\mathrm{GBS}^{14}$. Studies by McKhann GM et al and Morris $M$ et al also had GBS as the most common cause $\mathrm{c}^{12,15}$. The obvious reasons for this could be 1) inclusion of AES and hence 2) increased no. of cases and 3) decreased percentage of GBS.

Among the children with AES with AFP, fever and weakness were associated with altered mental status (87\%), convulsions (73.9\%) and loss of consciousness (34.8\%) which is similar to studies by Singh RR et al. ${ }^{16}$ and Rayamajhi $A$ et al. ${ }^{17}$. Similar to our study, viral encephalitis was the most common cause (46\%) of acute febrile encephalopathy in the study by Singh RR et al. ${ }^{16}$. Our study had smaller proportion of children (4.7\%) with Japanese encephalitis as compared to studies by Singh RR et al. $(40 \%)^{16}$, Rayamajhi A et al. $(61.7 \%)^{17}$ and Khinchi $Y$ R et al. $(18 \%)^{18}$. This could be due to the fact that they included all AES and not just AES with AFP. With the inclusion of JE vaccine in EPI schedule of Nepal as a part of routine immunization, the overall cases of JE have also decreased dramatically. In our study, only 1 (4.3\%) child had cerebral malaria with positive screening test in contrast to studies by Singh RR et al. and Kumar et al. ${ }^{16,20}$. Thus, it was an uncommon cause of fever presenting with encephalopathy in the present study area.

No virus was isolated from stool of the 20 children of AES with AFP and thus this strengthened the AFP surveillance. There are no published data of studies from Nepal for AES with inclusion of stool examination as per AFP surveillance guidelines till now. Inclusion of AES in AFP surveillance has been initiated in Nepal since 2004 so as not to miss any case of AFP.

In our study, Non-Polio Enterovirus (NPEV) was isolated in $4.3 \%$ children, of which all had complete recovery which is also similar to study in Pakistan by Saeed $\mathrm{M}$ et $\mathrm{al}^{30}$.

The most common electrolyte disturbance seen in our study was hypokalemia in $6.9 \%$ children, which is similar to study by Narang et al. ${ }^{10}$ and Sharma PP et al. ${ }^{29}$, where there were six malnourished children presenting with AFP due to hypokalaemia who were successfully treated with potassium supplementation emphasizing the need to consider hypokalaemia in the differential diagnosis of AFP in malnourished children.

In our study, $37.2 \%$ of cases were between one to five years with the mean age of $5.6 \pm 4.1$ years, which is similar to previous studies of Jalal et al, Bassey et al and Narang et $\mathrm{al}^{8,9,10}$. In our study, the male:female ratio was 1.39:1 which is also in accordance with the observations of other workers like Karmarkar SA et al. ${ }^{11}$ and Narang et al. ${ }^{10}$ which may be due to the fact that more male sick children are brought to hospitals in our country.

Among the children with GBS, preceeding viral illness was found in $75 \%$ children in form of diarrhea and respiratory tract infection similar to the study of Sharma KS et al. ${ }^{14}$ and other studies from Italy ${ }^{24}$, Sweden ${ }^{25}$, Srilanka ${ }^{26}$, Netherlands ${ }^{27}$. Complications of GBS were noticed in $50 \%$ children in the form of respiratory failure (50\%) and autonomic failure (25\%) with improvement seen in $25 \%$, which is comparable to studies by Sharma KS et al. ${ }^{14}$, Chang Q et al ${ }^{25}$, Narang et $\mathrm{al}^{10}$, Koul et $\mathrm{al}^{28}$ and Morris et $\mathrm{al}^{13}$.

The mean Glasgow Coma Scale in our study was $9.53 \pm 4.27$. There was significant difference in the mean GCS of survivor and non-survivor groups $(p=0.02)$. More than half $(65.2 \%)$ of the children with AES with AFP had GCS in the range of 3-8. Our finding is similar to the study by Singh RR et al, G Anga et al, CR Kennedy et al and Rayamajhi $A$ et al $^{16,21,22,23}$.

Overall, in our study, we found different causes with various clinico-epidemiological features for children presenting with AFP. AES was the major differential diagnosis, besides the known differential of Guillain Barre Syndrome and other causes. Various parameters like low GCS, high temperature, low respiratory rate, need for endotracheal intubation/ mechanical ventilation/ICU care, requirement of inotropes and complications like respiratory failure and autonomic failure were found to be significantly related to poor prognosis.

\section{Conclusion}

In our study, AES was one of the common causes of AFP and it could be the most common differential diagnosis, when all AES presenting with AFP were included. The differential diagnoses of AFP were found to be AES, GBS, dyselectrolytemia, peripheral neuritis, Non-polio Enterovirus and others like 
Neurocysticercosis, temporal lobe abscess,etc. Apart from floppiness/ weakness, the other associated presenting features of the typical disease supported to clinch the diagnosis. Low GCS, high temperature, low respiratory rate, need for endotracheal intubation/ mechanical ventilation/ICU care, requirement of inotropes and complications like respiratory failure and autonomic failure were associated with significant morbidity and mortality.

Acknowledgements: We would like to thank Dr. Rabindra Karna, Surveillance Medical Officer, WHOIPD and his entire team who helped to carry out AFP surveillance; Mr. D.D. Baral, Biostatistician, who helped with statistical analysis; and all our patients and their parents/guardians who participated and made this study successful.

\section{Funding: None}

Conflict of interest: This article is based on the final thesis (part of compulsory thesis submission) submitted to Department of Paediatrics, BP Koirala Institute of Health Sciences, Dharan, Nepal.

Permission from IRB: Yes

\section{References}

1. MarxA, Glass JD, Sutter RW. Differential Diagnosis of Acute Flaccid Paralysis and Its Role in Poliomyelitis Surveillance. Epidemiol Rev 2000;22(2):298-316.

2. Growdon JH, Fink JS. Paralysis and movement disorder. In: Isselbacher KJ, Braunwald E, Wilson JD, eds. Harrison's principles of internal medicine. New York, NY: McGraw- Hill Book Company, 1994:115-25.

3. Field Guide for Surveillance of Vaccine Preventable Diseases 2010; Government of Nepal/ World Health Organization.

4. The Global Polio Eradication Initiative 2010 (http://www.polioeradication.org/Mediaroom/ NewsletterPolioNews.aspx/).

5. World Health Organization/mediacentre/factsheet N114 November 2010 (http://www.who.int/ entity/mediacentre/en);UNICEF Nepal.

6. Joint National/International Review of Acute Flaccid Paralysis (AFP) Surveillance - Nepal25 March - 6 April 2006 and 6-12 August 2006.

7. Joint national International VPD Surveillance \& EPI Review, Nepal- review report 2010.

8. Jalal Poorolajal, Shadi Ghasemi, Leila Nezamabadi Farahani et al. Evaluation of Acute Flaccid Paralysis in Hamadan, Iran from 2002 to 2009. Epidemiol Health 2011;33:e2011011.

9. Bassey Enya Bassey, Alex Gasasira, Pamela Mitula, Umoh Utobong Frankson, Johnson Adekunle Adeniji. Surveillance of acute flaccid paralysis in Akwa Ibom State, Nigeria 2004-2009. Pan African Med J 2011;9:32.

10. Narang G S, Pahwa J S. Retrospective Study of Acute Flaccid Paralysis Cases from a Tertiary Care Centre in Amritsar. Pediatric Oncall 2011; 8. Art \# 14. (http://www.pediatriconcall.com/fordoctor/ Medical_original_articles/flaccid_paralysis.asp).

11. Karmarkar SA et al. A study of acute febrile encephalopathy with special reference to viral etiology. Indian J Pediatr 2008;75(8):801-5. Epub 2008 Sep 4.

12. McKhann GM, Cornblath DR, Griffin JW, et al. Acute motor axonal neuropathy: a frequent cause of acute flaccid paralysis in China. Ann Neural 1993;33:333-42.

13. Morris AMS, Elliott EJ, Antony J et al. Acute flaccid paralysis in Australian children. J Paediatr Child Health 2003;39(1):22-26

14. Sharma KS, Singh R, Shah GS. Guillain Barre Syndrome; Major Cause of Acute Flaccid Paralysis in Children and Adolescents of Nepal. J Nep Paedtr Soc 2011;31(2):93-97.

15. Andrus JK, de Quadros CA, Olive JM. The surveillance challenge: final stages of eradication of poliomyelitis in the Americas. MMWR CDC Surveill Summ 1992;41:21-6.

16. Singh RR et al. Clinical and etiological profile of acute febrile encephalopathy in eastern Nepal. Indian J Pediatr 2009;76(11):1109-11.

17. Rayamajhi A, Singh R, Prasad R, Khanal B, Singhi S. Study of Japanese encephalitis and other viral encephalitis in Nepali children. Pediatr Int 2007;49(6):978-84.

18. Y. R.Khinchi, A. Kumar, S. Yadav. Study of acute encephalitis syndrome in children. J Coll Med Sci 2010;6(1):7-13

19. Rayamajhi A, Singh R, Prasad R, Khanal B, Singhi $\mathrm{S}$. Clinico-laboratory profile and outcome of Japanese encephalitis in Nepali children. Ann Trop Paediatr 2006;26(4):293-301.

20. Kumar R, Mathur A, Kumar A, Sethi GD, Sharma S, Chaturvedi UC. Virological investigations of acute encephalopathy in India. Arch Dis Child 1990; 65: 1227-1230. 
21. Anga G, et al. The aetiology, clinical presentations and outcome of febrile encephalopathy in children in Papua New Guinea. Ann Trop Paediatr 2010;30(2):109-18.

22. C R Kennedy, S W Duffy, R Smith, R 0 Robinson. Clinical predictors of outcome in encephalitis. Arch Dis Childhood 1987; 62:1156-162

23. Rayamajhi A, et al. Clinical and prognostic features among children with acute encephalitis syndrome in Nepal; a retrospective study. BMC Infect Dis. 2011; 28(11):294.

24. Emilia-Romagna study group on clinical and epidemiological problems in Neurology. A prospective study on the incidence and prognosis of Guillain Barre Syndrome in EmiliaRomagna region, Italy (1992-1993). Neurology 1997;48(1):214-221.

25. Cheng $Q$, Jiang GX, Press $R$, Andersson $M$, Ekstedt $B$, Vrethem et.al. Clinical epidemiology of GBS in adults in Sweden 1996-1997: A prospective study. Eur J Neurol 2000;7(6):685-692.
26. Seneviratne U. Guillain Barre Syndrome. Postgrad Med J 2000;76:774-782.

27. Jacobs $B C$, Rothbarth $P H$, van der Meché FG, Herbrink P, Schmitz PI, de Klerk MA, van Doorn PA. The spectrum of antecedent infections in GBS: A case-control study. Neurology 1998;51(4):1110115.

28. Koul R, Al-Futaisi A, Chacko A, Fazalullah M, Nabhani SA, Al-Awaidy S, Al-Busaidy S, Al-Mahrooqi S. Clinical characteristics of childhood guillain-barré syndrome. Oman Med J 2008;23(3):158-61.

29. Sharma PP, Murali MV, Koul PB, Dhar P. Hypokalaemic paralysis in malnourished children. Ann Trop Paediatr 1992;12(2):173-5

30. Mohsan Saeed, Sohail Z Zaidi, Asif Naeem, et al. Epidemiology and clinical findings associated with enteroviral acute flaccid paralysis in Pakistan. BMC Infectious Diseases 2007, 7:6 doi:10.1186/14712334-7-6 (http://www.biomedcentral.com/14712334/7/6). 